\title{
Phenotypic and Differentiation Stability of Human Embryonic Stem Cell-Derived Osteoblasts
}

\author{
Premjit Arpornmaeklong ${ }^{a, b}$ Michael J. Pressler ${ }^{b}$ Paul H. Krebsbach ${ }^{b}$ \\ ${ }^{a}$ Department of Oral and Maxillofacial Surgery, School of Dentistry, Prince of Songkla University, Hat Yai, Thailand; \\ ${ }^{b}$ Department of Biologic and Materials Sciences, School of Dentistry, University of Michigan, Ann Arbor, Mich., USA
}

\section{Key Words}

Human embryonic stem cells • Osteoblastic differentiation • Tumorigenesis • Transdifferentiation

\begin{abstract}
Purpose: To ensure the efficiency and safety of transplanted human embryonic stem cell (hESC)-derived osteoblast-like cells (hESC-OS) for bone regeneration, this study was designed to determine the effects of continuous cell expansion on the osteoblastic differentiation stability, pluripotency, and tumorigenic potential of long-term expanded hESC-OS. Methods: hESCs manually harvested as cell aggregates or enzymatically dissociated as single cells were directly incubated in osteogenic medium and serially passaged to passage 25. Expression of osteoblast-related genes, pluripotent regulator genes, and genes related to tumorigenesis were examined at the primary passage and every 5 passages thereafter. hESC-OS were subcutaneously transplanted into nude mice for 4-24 weeks to test for teratoma formation. hESC-OS were recultivated in hESC culture conditions to evaluate the extent to which reverse differentiation back to the undifferentiated stage may occur. Results: hESC-OS derived from $\mathrm{hESC}$ aggregates and dissociated cells exhibited comparable osteoblast differentiation patterns. Expression levels of osteoblast-related genes reached plateau levels at passages $5-10$ before declining in higher passages. Expres-
\end{abstract}

\section{KARGER}

Fax +4161306 1234

E-Mail karger@karger.ch

www.karger.com (c) 2011 S. Karger AG, Basel

Accessible online at: www.karger.com/cto sion of tumor-associated genes was not significantly increased. Only hESC-OS at primary and first passages formed teratomas after 4 weeks in vivo. The hESC-OS were not able to revert to hESCs. Conclusions: Expanded hESC-OS demonstrated lineage-specific differentiation stability, did not maintain the pluripotency of hES cells, and were genetically stable. Thus, hESC-OS may be considered for large animal preclinical studies.

Copyright $\odot 2011$ S. Karger AG, Basel

\section{Introduction}

To generate the number of osteogenic cells derived from human embryonic stem cells (hESCs) required for cell-based regeneration, a number of challenges must be overcome. Among these challenges is the necessity to

\section{Abbreviations used in this paper}

hESC-OS osteoblast-like cells derived from hESCs in osteogenic medium

hESCs human embryonic stem cells

hMSCs human mesenchymal stem cells

qRT-PCR quantitative real-time reverse transcriptase polymerase chain reaction
Dr. Paul H. Krebsbach, Department of Biologic and Materials Sciences

University of Michigan School of Dentistry

1011 North University Avenue, Rm 1030 Kellogg

Ann Arbor, MI 48109-1078 (USA)

Tel. +1 734936 2600,E-Mail paulk@umich.edu 
Table 1. List of primers used in the qRT-PCR analysis

\begin{tabular}{lll}
\hline Names/symbols & Gene description & Catalog numbers \\
\hline ALPL & alkaline phosphatase, liver/bone/kidney & Hs01029141_g1 \\
Beta-actin (ACTB) & human ACTB (actin, beta) endogenous control & $4333762 T$ \\
hRas & v-Ha-ras Harvey rat sarcoma viral oncogene homolog & Hs00610483_m1 \\
Myc (c-Myc) & v-myc myelocytomatosis viral oncogene homolog & Hs99999003_m1 \\
Nanog & Nanog homeobox & Hs02387400_g1 \\
Oct4 (POU5F1) & POU class 5 homeobox 1 & Hs03005111_g1 \\
Osteocalcin (BGLAP) & bone gamma-carboxyglutamate (gla) protein & Hs01587814_g1 \\
Runx2 & Runt-related transcription factor 2 & Hs00231692_m1 \\
SNRPE (bRaf) & small nuclear ribonucleoprotein polypeptide E & Hs01635040_s1 \\
Sox2 & SRY (sex determining region Y)-box & Hs01053049_s1 \\
\hline
\end{tabular}

prolong the expansion of the differentiated cells before transplantation and to ensure transplantation of homogenous and lineage-restricted cells.

Long-term in vitro cultures of human mesenchymal stem cells (hMSCs) and osteoprogenitor cells are associated with reduced proliferative and differentiation potential due to cellular senescence [Bruder et al., 1997; Kim et al., 2009]. Furthermore, some reports have indicated that long-term culture may cause a spontaneous transformation of hMSCs [Rubio et al., 2005; Rosland et al., 2009]. This transformation potential is amplified when the differentiated cells are derived from pluripotent cells such as hES cells that are known to have a high capacity to form teratomas in vivo [Takahashi and Yamanaka, 2006].

Therefore, to ensure the efficiency and safety of hESCderived osteoblast-like cells (hESC-OS), the goals of this study were: (1) to determine the effects of continuous cell expansion on osteoblastic differentiation stability and (2) to examine the pluripotency and tumorigenic potential of long-term expanded hESC-OS.

\section{Materials and Methods}

\section{Human Embryonic Stem Cell Culture and Osteogenic} Induction

Undifferentiated hESCs (BG01; Bresagen, Inc., USA) (passages 35-50) as (1) a suspension of single cells or (2) cell aggregates were regularly passaged in osteogenic medium as described [Arpornmaeklong et al., 2010]. A karyotype analysis was performed by cytogenetic analysis on $20 \mathrm{G}$-banded metaphase cells of hESCsOS at passages 5, 10, 17, and 20 (Cell Line Genetics, USA).

\section{Quantitative Real-Time Reverse Transcriptase Polymerase}

Chain Reaction

Quantitative real-time reverse transcriptase polymerase chain reaction (qRT-PCR) was performed on hESCs and hESC-OS at the primary passage and every 5 passages thereafter as described
[Arpornmaeklong et al., 2010]. The genes and primers used are listed in table 1.

\section{Reversion of Differentiation}

hESC-OS at passages 1,5 , and 10 were seeded in 6-well culture plates $\left(5 \times 10^{3}\right.$ cells $\left./ \mathrm{cm}^{2}\right)$ and incubated for 21 days in hESC medium, human fetal fibroblast condition medium (Stemcell Technologies, Canada) on a Matrigel- (BD Biosciences, USA) coated surface for hESC culture, and osteogenic mediums (as described in the previous section) on a cell culture plate surface for differentiation culture. Cell morphology and undifferentiated cell colony formation were observed at the time of cell culture change. On day 21, hESC-OS were collected for expression of pluripotency related genes Oct4, Nanog, Sox2, and Lin 28 (table 1) using qRTPCR analysis [Takahashi and Yamanaka, 2006].

\section{Preparation of Composite Scaffolds and Subcutaneous}

Transplantation

All procedures were approved by the University of Michigan Committee on the Use and Care of Animals. On culture days 6 and 7, undifferentiated hESCs (BGO1) at passages 50-60 were dissociated and hESC-OS at passages $1,5,10,15,20$, and 25 were trypsinized. Cell- $\left(2 \times 10^{6}\right.$ cells/pellet $)$ gelatin sponge- $(7 \times 3 \mathrm{~mm}$; Pharmacia \& Upjohn Co., USA) fibrin gel [40 $\mathrm{mg} / \mathrm{ml}$ fibrinogen with 200 units/ml human thrombin (Sigma, USA)] composite scaffolds were prepared as described [Arpornmaeklong et al., 2010]. Six composite scaffolds were prepared for the transplantation of hESCs at passages 50-60 and hESC-OS at passage 1 . A total of 18 composite scaffolds were prepared for the transplantation of dissociates and aggregates of hESC-OS at passages 5-25. Composite scaffolds were subcutaneously transplanted in 30 immunocompromised mice (N:NIHbg-nu-xid; Harlan Laboratories, Inc., Indianapolis, Ind., USA), with 4 pockets in 1 mouse and 1 sample in 1 pocket. Each pocket on 1 animal contained a composite scaffold with cells from different passages. The transplants were harvested 4,12 , and 24 weeks after transplantation. Histological sections were examined for tumor formation [Takahashi and Yamanaka, 2006].

Statistical Analysis

Results were derived from 3 strains each of hESCs cultured as dissociated cells and cell aggregates. Data were analyzed by 1-way analysis of variance (ANOVA) at $\mathrm{p}<0.05$. 


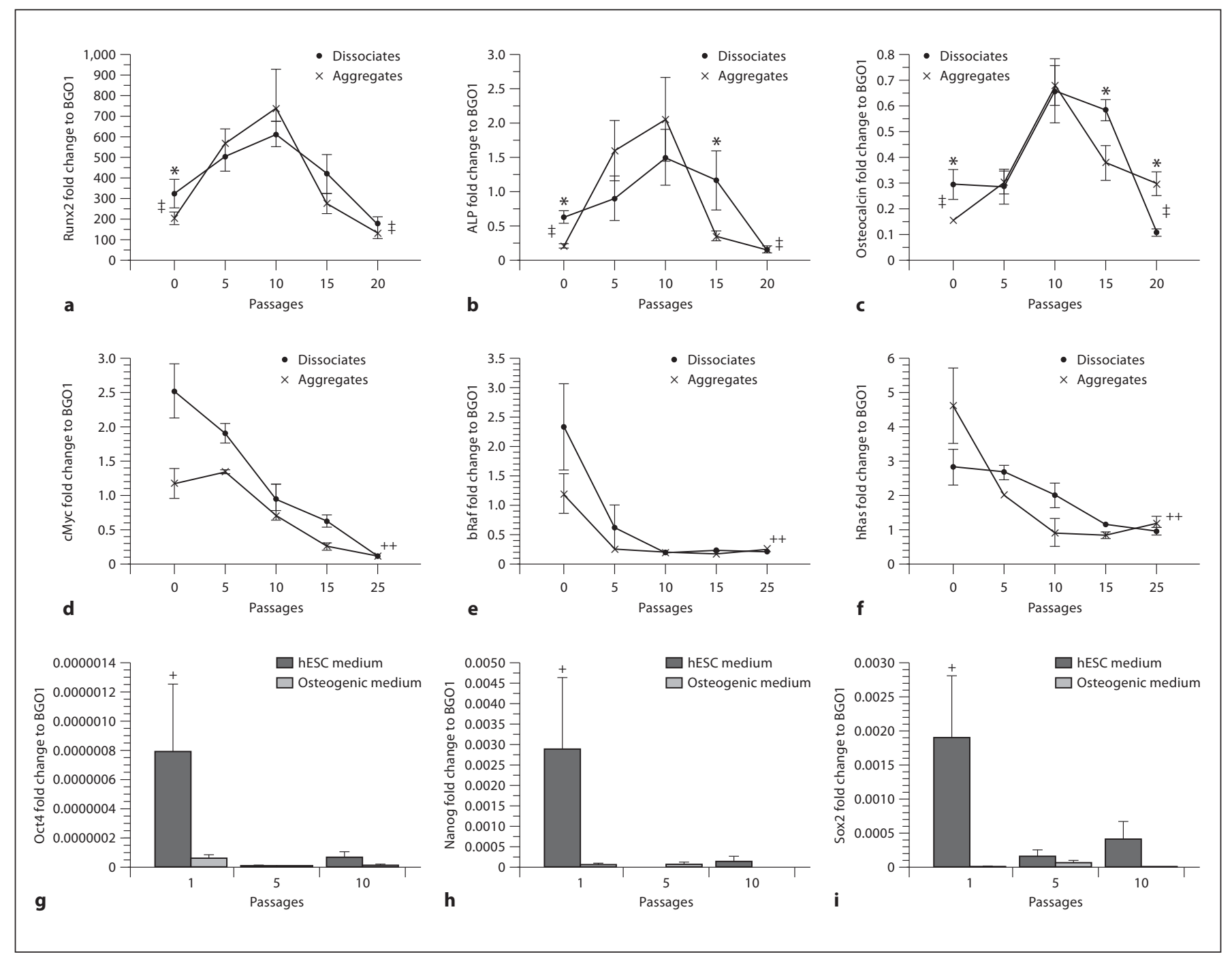

Fig. 1. hESC-OS showed osteogenic differentiation potential and genotypic stability by exhibiting an absence of spontaneous tumorigenic differentiation. Expression profiles of osteoblast-related genes $(n=3)(\mathbf{a}-\mathbf{c})$ and oncogenes $(\mathbf{d}-\mathbf{f})$ over 20 cell passages in osteogenic medium $(n=3)$. $\mathbf{g}-\mathbf{i}$ Decrease in the expression levels of pluripotency regulator genes at passages 5 and 10 following a reverse differentiation study $(\mathrm{n}=6)$. g Oct4. $\mathbf{h}$ Nanog. $\mathbf{i}$ Sox $2 .{ }^{*}=$ Significant differences between groups at the same passages; $\neq=$ lower levels than passage $10 ;++=$ significantly lower levels than the primary passage (passage 0$) ;+=$ highest expression levels (mean \pm SEM, $\mathrm{p}<0.05$ ).

\section{Results and Discussion}

The decrease in osteoblastic differentiation potential after the extensive expansion of hESC-OS in vitro (fig. 1ac) is similar to those of osteoblast-like cells derived from hBMSCs [Bruder et al., 1997] and hMSCs [Kim et al., 2009] in aging or presenescent stages.

Tumorigenic transformation of the differentiated cells is another major concern for safe clinical application when using differentiated cells derived from stem cells. Transformed mesenchymal stem cells have a significantly increased proliferation rate, altered morphology and phenotype, and form tumors in vivo [Rubio et al., 2005; Rosland et al., 2009]. In the current study, the phenotypic and genotypic stability of hESC-OS in long-term expansion was indicated by the consistent absence of chromosomal abnormalities of hESC-OS at passages 5-25 revealed by normal karyotypes (data not shown), decreased 

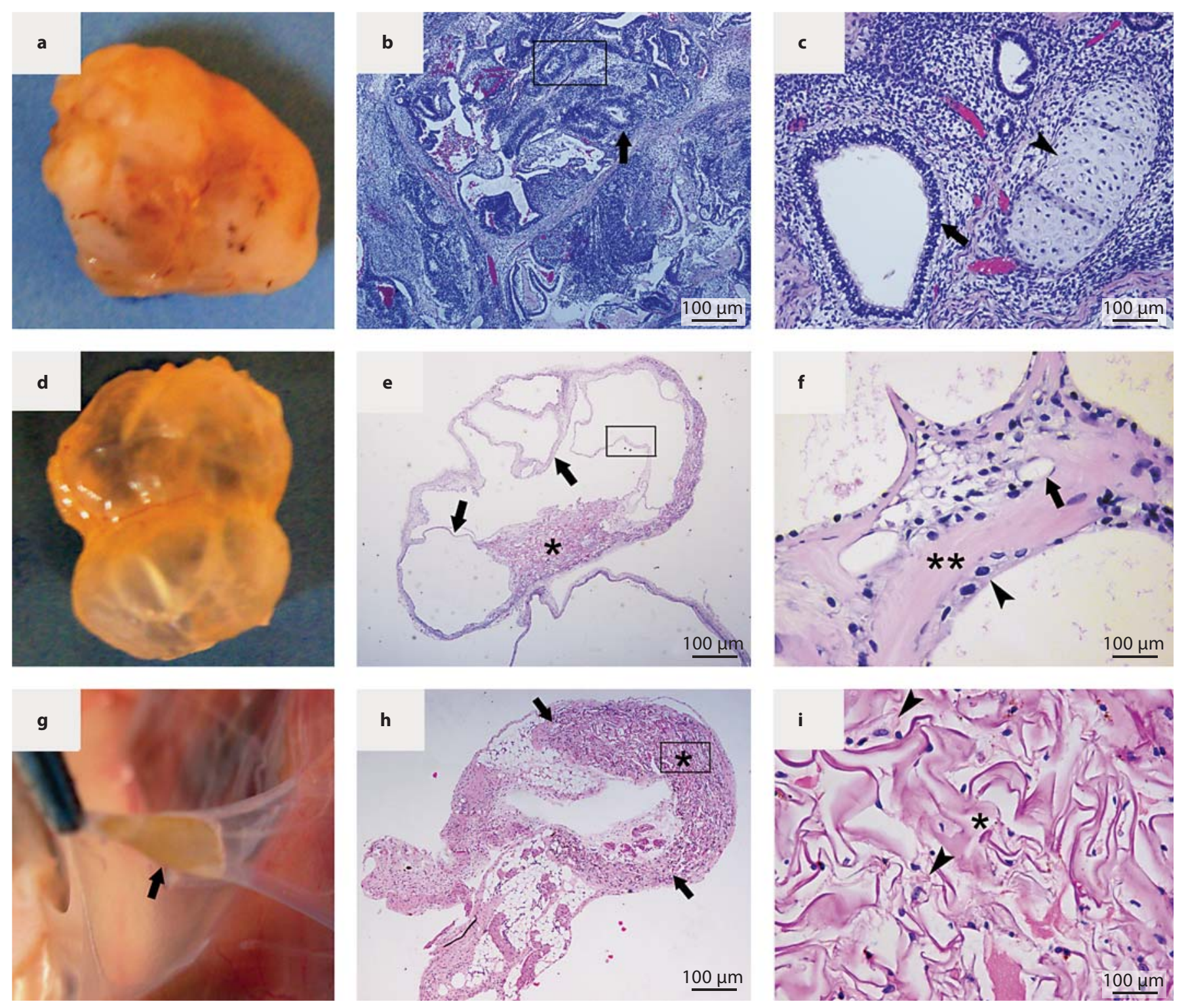

Fig. 2. Absence of tumorigenicity and undifferentiated cells from long-term transplanted hESC-OS. Transplantation of hESCs (ac) and hESC-OS (d-f) at passage 1 for 4 weeks and a representative of expanded hESC-OS (g-i) at passage 10 for 24 weeks. The insets indicate selected regions of interest. The magnified images from the selected regions of interest are shown in the subsequent images $(\mathbf{c}, \mathbf{f}, \mathbf{i})$. a Solid tumor from transplanted hESCs. b, c Histology of solid tumor revealing predominantly ductal tissue from the endodermal lineage, ductal tissue (arrow), and cartilage (arrowhead). d Cystic lesion containing clear fluid from the transplanta- tion of hESC-OS at passage 1. e Revealing cystic lesion with multiple compartments of thin fibrous tissue wall (arrow) and remnants of the gelatin sponge $\left(^{*}\right)$. $\mathbf{f}$ Cystic wall comprised of collagenous stroma ${ }^{* *}$ ), blood vessel (arrow) and epithelium cells (arrowhead). $\mathbf{g}$ Remnant of an hESC-OS-gelatin sponge construct 24 weeks after transplantation. h Approximate margins (arrows) of a remnant gelatin sponge $\left(^{*}\right)$ embedded in host connective and fat tissues. i Gelatin sponge $(*)$ infiltrated with connective tissue (arrowheads) with no evidence of teratoma formation 24 weeks after transplantation. 
expression levels of oncogenes (fig. 1d-f), the lack of dedifferentiation (fig. 1g-i), and the absence of tumor formation after long-term transplantation (fig. 2). Moreover, an absence of undifferentiated colonies in the reversionof-differentiation study further supported a lack of pluripotent cells and lack of undifferentiated cells in the hESC-OS (data not show) [Takahashi and Yamanaka, 2006].

The absence of tumor formation from long-term transplantation of expanded hESC-OS up to passage 25 further substantiated genomic stability and the absence of undifferentiated cells in the expanded hESC-OS population. Solid tumor and cystic lesion formation were found 4-6 weeks after transplantation in all samples of hESCs (fig. $2 \mathrm{a}-\mathrm{c}$ ) and hESC-OS at passage 1, respectively (fig. $2 \mathrm{~d}-\mathrm{f})$. All expanded hESC-OS at passages 5 and higher $(10,15,20$ and 25) did not form teratomas even after 6 months in vivo. Sixth months after implantation, the transplants were extensively resorbed and residual scaffolds were infiltrated with connective tissue (fig. $2 \mathrm{~g}-\mathrm{i}$ ).
Despite these findings, the alloimmunogenic reaction and integration of differentiated cells into a local environment and continuous growth and function as differentiated cells are the principle challenges requiring further studies before hESC-OS can be used for clinical applications [Motaln et al., 2010].

Taken together, expanded hESC-OS acted as normal somatic tissue with a limited life span. Our data suggest that the application of hESC-OS at passages 5-10 should be used for developmental studies and transplantation. The current study supports the safe use of hESC-OS in in vivo applications and thus hESC-OS may be considered for large animal preclinical studies.

\section{Acknowledgements}

This work was supported by NIH/NIDR RO1DE 016530 (to P.H.K.). The authors thank the University of Michigan Stem Cell Core for support and Dr. Luis G. Villa-Diaz for advice.

\section{References}

Arpornmaeklong, P., Z. Wang, M.J. Pressler, S.E. Brown, P.H. Krebsbach (2010) Expansion and characterization of human embryonic stem cell-derived osteoblast-like cells. Cell Reprogram 12: 377-389.

Bruder, S.P., N. Jaiswal, S.E. Haynesworth (1997) Growth kinetics, self-renewal, and the osteogenic potential of purified human mesenchymal stem cells during extensive subcultivation and following cryopreservation. J Cell Biochem 64: 278-294.

Kim, J., J.W. Kang, J.H. Park, Y. Choi, K.S. Choi, K.D. Park, D.H. Baek, S.K. Seong, H.K. Min, H.S. Kim (2009) Biological characterization of long-term cultured human mesenchymal stem cells. Arch Pharm Res 32: 117-126.
Krebsbach, P.H., S.A. Kuznetsov, K. Satomura, R.V. Emmons, D.W. Rowe, P.G. Robey (1997) Bone formation in vivo: comparison of osteogenesis by transplanted mouse and human marrow stromal fibroblasts. Transplantation 63: 1059-1069.

Motaln, H., C. Schichor, T.T. Lah (2010) Human mesenchymal stem cells and their use in cellbased therapies. Cancer 116: 2519-2530.
Rosland, G.V., A. Svendsen, A. Torsvik, E. Sobala, E. McCormack, H. Immervoll, J. Mysliwietz, J.C. Tonn, R. Goldbrunner, P.E. Lonning, R. Bjerkvig, C. Schichor (2009) Longterm cultures of bone marrow-derived human mesenchymal stem cells frequently undergo spontaneous malignant transformation. Cancer Res 69: 5331-5339.

Rubio, D., J. Garcia-Castro, M.C. Martin, R. de la Fuente, J.C. Cigudosa, A.C. Lloyd, A. Bernad (2005) Spontaneous human adult stem cell transformation. Cancer Res 65: 30353039.

Takahashi, K., S. Yamanaka (2006) Induction of pluripotent stem cells from mouse embryonic and adult fibroblast cultures by defined factors. Cell 126: 663-676. 